\title{
Towards Better Fitting Data Warehouse Systems
}

\author{
Naveen Prakash, Deepika Prakash, and Y.K. Sharma \\ MRCE Faridabad, IIIT Banglore, NIC, New Delhi, INDIA \\ praknav@hotmail.com, dpka.prakash@gmail.com, yks@nic.in
}

\begin{abstract}
In order to produce data warehouse systems that reflect organizational decisional needs, development should be rooted in the goals and decisions of organizations. The goal-decision-information model and associated information elicitation techniques for decision making are presented. There are four main techniques, Ends analysis, Means analysis, Critical Success Factor analysis, and Outcome Feedback analysis. Using these, the requirements engineer is able to elicit the required information as well as the sub decisions of a given decision. The elicitation techniques are then applied to these sub decisions. The elicitation process ends when all decisions/sub decisions have been thus processed. A comparison of this approach is made with data base driven and ER driven development approaches to data warehouse development to show that it produces systems that fit well with decisional requirements.
\end{abstract}

Keywords: Data Warehouse system, requirement engineering.

\section{Introduction}

Enterprise information systems come to us in many forms ranging from early 'packages' like payroll systems to those addressing application domains like hotel reservation systems, full enterprises like ERP systems and through to systems supporting cross organization operations like supply chain management. Notice that this evolution considers the class of functional/transactional systems. However, there is another way of looking at enterprises, not in terms of what they do but in terms of the decisions that they have to make. In this approach, information generated as a trace of functions/transactions carried out is not interesting per se. Rather it is the management decisions that need to be taken to change enterprise operation that comes to the fore. Therefore, now information is a record of what happened and it can be analysed to reveal trends, patterns, associations etc. that can be used to take decisions. This is the area of enterprise information systems as supported by Data Warehouse (DW) technology and we shall refer to these as decisional enterprise systems in contrast to the other functional enterprise systems.

Decisional enterprise systems share a number of common problems with the functional class. An important one is the issue of fitment: can we ensure that the decisions made in organizations are indeed supported by the information contained in the data warehouse? Is the Data warehouse a faithful representation of the information that is to be kept in the warehouse? In other words, the problem is of building data warehouses whose content fits well with information needed for decision making. 
In this paper, we will show that the Goal-Decision-Information or GDI approach [Pra04, Pra08] when supported by appropriate information elicitation technique promote a direct relationship between DW information contents and the decisions of interest. We propose four elicitation techniques, Ends analysis, Means analysis, Outcome Feedback analysis, and Critical Success Factor analysis. These techniques are applied to an initial, given set of decisions to determine decision parameters. An analysis of these parameters is then carried out to determine those which are sub decisions of the initial decision, and those which are information to be kept in the Warehouse. Elicitation is done for each sub decision so discovered. The elicitation process ends when no further decisions are to be processed.

This paper is organized in three main sections. The next section, Section II, considers the DW in the organizational context. It presents the GDI approach and the manner in which it can be supported to arrive at the information contents of the data warehouse to-be. Section III contains a comparison of the proposed technique with conventional data warehouse development life cycles. This comparison is based on experience of developing a data warehouse using database driven, ER driven, and GDI driven techniques respectively.

\section{DW in the Organizational Context}

In this section, we present a technique that arrives at DW information contents through an exploration of organizational goals, associated decisions to meet these, and information relevant to decision making.

\subsection{The Goal-Decision-Information (GDI) Model}

The basic assumption of the GDI model is that since a data warehouse system is used for decision-making, any model that is developed must be rooted in the essential nature of decision-making. According to [Mon86] the decision-making process consists of three phases, (a) intelligence, that involves searching for conditions that call for decisions, (b) design, which involves inventing, developing, and analyzing possible courses of actions, and (c) choice, which implies the selection of a course of action from those available.

We model [Pra08] the notion of 'conditions that call for decisions' through the concept of a goal. We view a goal as an aim or objective that is to be met. A goal is a passive concept and it cannot perform or cause any action to be performed; it is nonoperational. As shown in Fig.1, a goal can be either simple or complex. A simple goal cannot be decomposed into simpler one. A complex goal is built out of other goals which may itself be simple or complex. The component goals of a complex one may be in an AND/OR relationship with one another. This is in accordance with goal modelling approaches of traditional Requirements engineering.

The Requirements engineering view [Pra08] suggests that goals 'guide decisions'. So, we naturally get the second concept of the GDI model, that of a Decision. For us, a decision is a specification of an active component that causes goal fulfillment. As shown in Fig.1, a decision can be either simple or complex. A simple decision cannot 


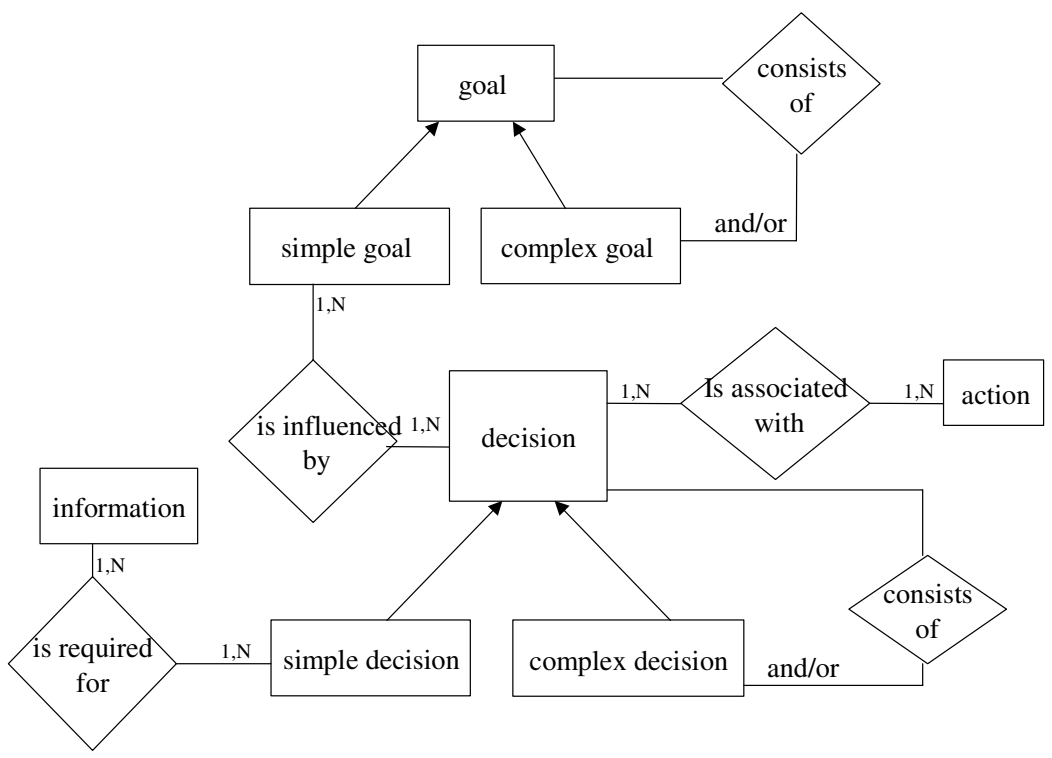

Fig. 1. The GDI Model (taken from [Pra08])

be decomposed into simpler ones whereas a complex decision is built out of other simple or complex decisions. Fig. 1 also shows that there is a relationship between goals and decisions through the association 'is influenced by' between goals and decisions. This association identifies the decisions which when taken can lead to goal satisfaction.

According to [Mar91] information is the critical resource for decision-making. This is captured, in Fig. 1, by the association is required for between decisions and decisional information. The is required for ensures that only relevant information is associated with decisions.

Notice the strong embedding of the GDI diagram in the larger decision making environment of an organization. For a decision-maker the primary task is to take the appropriate decision and obtaining information is the means to do it. Thus, the GDI diagram-decision-maker interaction is done in terms of the goals, decisions, and information that the decision makers see and work with. The decisions and information here are necessarily high level and a number of details remain hidden. For example, one may only identify Market Profile as the information needed without making it precise, what comprises it? How does it translate to the data structure to be kept in the Data Warehouse? How shall it be physically organized? This is in consonance with our desire to establish a broad alignment between organizational needs for decision making information and a specification of what shall be kept in the DW.

The GDI diagram is obtained by instantiating the GDI model with the relevant goals, decisions, information and relationships between these. As an example, consider a vehicle manufacturing company that has one of its goals as Provide Responsible After Sales Service. Now, an important decision that influences this goal is to Recall Product and requires information about the suppliers of the company, the material 
supplied by them, customer complaints, customer data, inspection reports of faulty products etc. This goal-decision-information data is used to instantiate the GDI model.

\subsection{Eliciting Information}

The GDI diagram suggests a three stage elicitation process for goals, decisions and information respectively as shown in Fig. 2.

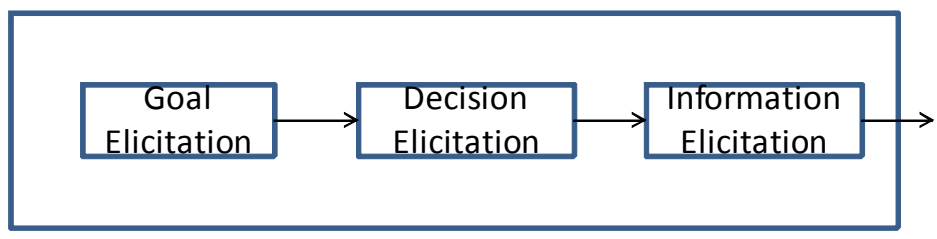

Fig. 2. The Three Stage Goal-Decision-Information Process

Our interest in this paper is in the information elicitation stage that addresses the problem, given a set of decisions elicit information relevant to these.

We introduce our elicitation technique by describing a tool called Raju whose architecture is shown in Fig. 3. There are four main components, Decision Parameter Guide, Information Identifier, Decision Identifier and the Verifier. All these interact with the repository of Raju. Conceptually, this repository is organized in three parts. The first part contains the goals of the organization, the second contains the set of decisions, and the third contains the parameters of the decisions. These parts are related to one another. The Goal and Decision parts are linked together by the GoalDecision relationship to keep track of which goal is satisfied by which decision. Similarly, the Decision and Parameter parts are linked together to keep a record of which parameter is relevant to which decision.

The four main components of Raju carry out specific functions as follows:

Decision Parameter Guide (DPG): This component is organized in four parts corresponding to the elicitation strategies of Critical Success Factor, Ends, Means and Outcome Feedback analysis respectively. The engineer is offered a menu of these and is thereafter guided to enter the material required by the selected strategy. As we shall see later, each strategy culminates in identifying the parameters of relevance to the decision being explored. Decision parameters can be interpreted either as sub decisions of the decision or as information relevant to the decision. Once the parameter of decision is identified then this distinction can be made by going to the Information Identifier.

Information Identifier: This component supports the application of heuristics that determine whether a given parameter is to be interpreted as information or not. These heuristics identify a number of properties of parameters and if the parameter possesses any one or more of these, then the parameter is treated as information otherwise not. 


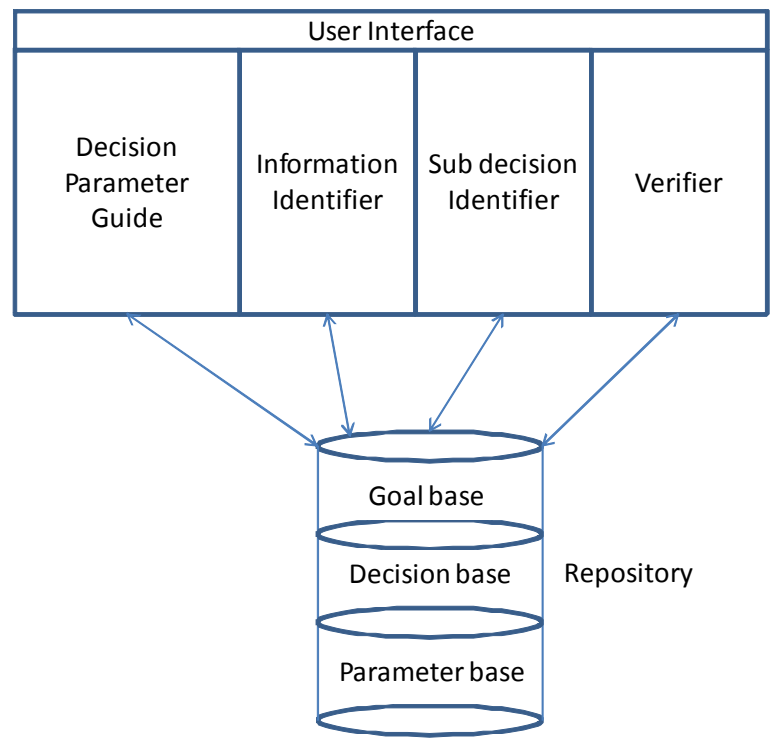

Fig. 3. The Architecture of Raju

Sub Decision Identifier: As its name implies, the Decision Identifier processes parameters to identify which of these are to be treated as sub decisions.

Verifier: The verifier looks to see that

- All initial decisions and those produced by the Decision Identifier have gone through the requirements engineering process,

- All parameters of all decisions have been identified as either information or decisions.

We briefly review the elicitation techniques available in Raju. These are Critical Success Factor (CSF) Analysis, Ends Analysis, Means Analysis, and Outcome Feedback Analysis.

\subsubsection{CSF Analysis}

According to (Wet91) most managers have a portfolio of 4-8 critical factors on which their success or failure depends. The essential question here is to identify the parameters that must be monitored to ensure that these factors remain in control. This control is carried out by appropriate decision making. Therefore, CSF analysis provides structure (Mon86) to the decision-maker/requirements engineer interaction thereby reducing the chance of including irrelevant data in the decisional system.

CSF Analysis is a three step process consisting of (a) CSF determination, (b) determination of CSF parameters, and (c) determination of properties of parameters. CSF analysis for a decision is complete when all its CSFs have been determined and steps (b) and (c) have been carried out for each of these 
Goal Improve health care

Please choose one of the following

ค Already existing CSF

Add new CSF

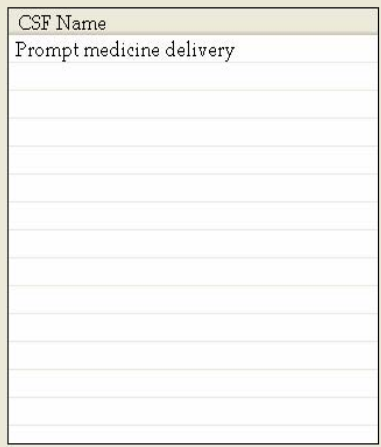

Decision Add new pharmacy

Update existing paramters

Insert new parameter set

\begin{tabular}{|c|c|c|c|c|c|}
\hline HA stant & Ddec_tree & Fo dec_tree (Runnin.... & 国 Gosal_Decision & 필 Document1 - Micr... & 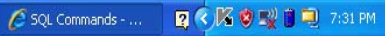 \\
\hline
\end{tabular}

Fig. 4. Obtaining CSF and its parameters

Raju provides the user interface shown in Fig. 4 for CSF elicitation. The top of the screen of Fig. 3, shows that requirements engineering for the decision Add new Pharmacy for the goal Improve health care is being done. The left hand side of the screen allows the requirements engineer to either enter the CSF affected by the decision or to select an existing CSF for modification. In the latter case, selection can be made from the list displayed.

The screen shows that a new CSF, Prompt medicine delivery, has been entered and that its parameter is waiting time of patient. In general, these may be more than one parameter for a given CSF.

\subsubsection{End Analysis}

The second elicitation process in Raju is Ends Analysis. 'Ends' refers to the result achieved by a decision. It identifies a concrete change in the organization that is a consequence of the decision. Ends analysis, is the identification of information needed to evaluate the effectiveness of the end to be achieved. The decision-maker/requirements engineer interaction is centred round determining the parameters of interest in estimating this effectiveness. As shown by (Wet91) for an order processing system, parameters 
required to evaluate the end "provide customer service" are, for example, customer credit status and payment history.

The needed parameters are again obtained in Raju by following a three step elicitation process. These steps are (i) determining Ends, (ii) determining the effectiveness measures of the Ends, and (iii) determining the parameters pertaining to evaluating the effectiveness. The relevant screen of Raju is shown in Fig. 5. Similar to the CSF analysis screen, on the left hand side of Fig. 4 Raju elicits the Ends of the decision. As before, an option exists to modify existing Ends. The right hand side of the screen contains boxes for entering the effectiveness measure as well as the parameters that help to evaluate the effectiveness measure.

The example shown in Fig.5 is for the same decision, Add new pharmacy, as before. An End of this decision is Full utilization of the new pharmacy, the effectiveness measure of this end is Service provided and one of the parameters of this measure is Amount of medicine distributed.

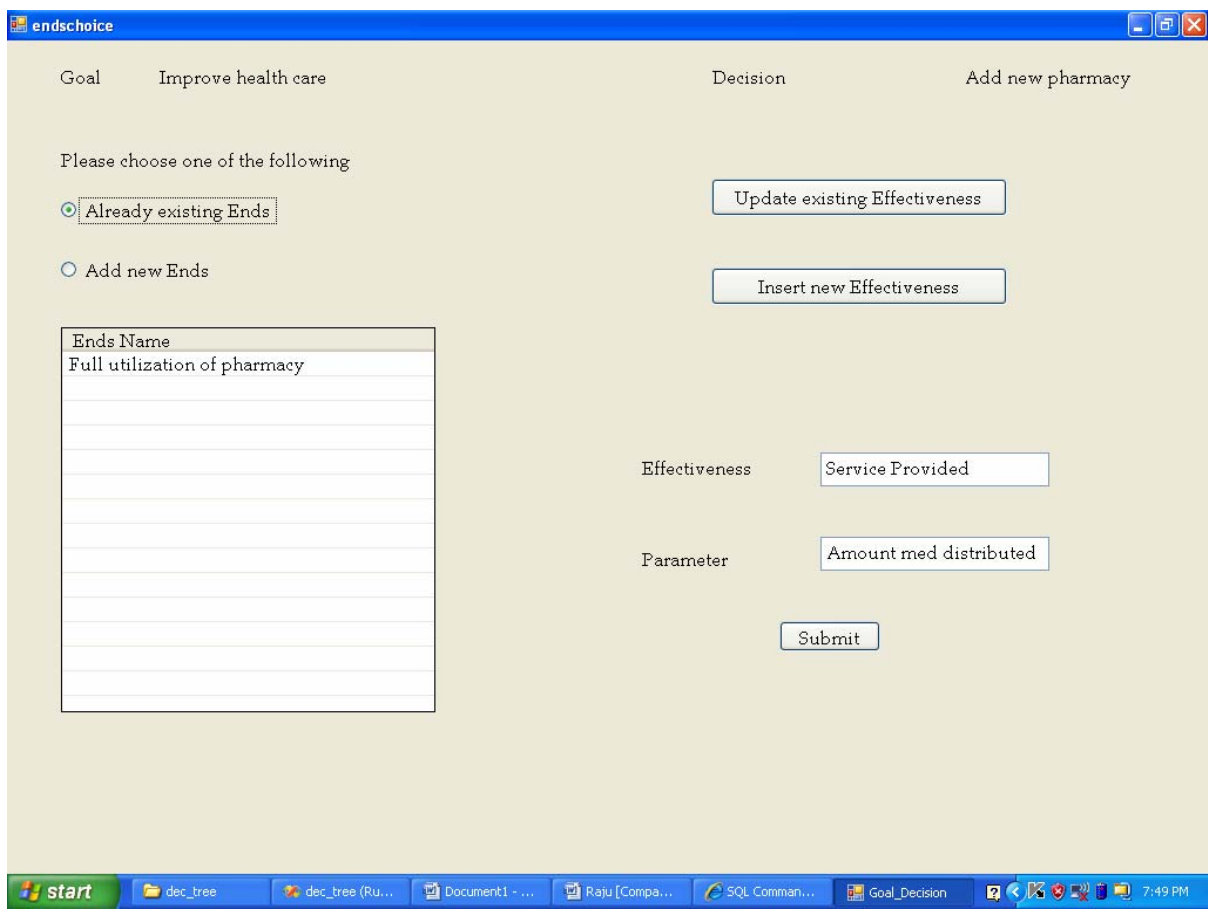

Fig. 5. Ends Analysis for Obtaining Decision Parameters

\subsubsection{Means Analysis}

Means analysis, is the identification of parameters needed to evaluate the efficiency of the means adopted to produce the ends. Thus, the requirements engineer/stakeholder interaction is now centered round eliciting parameters that provide information on the efficiency of the means adopted for each decision. 


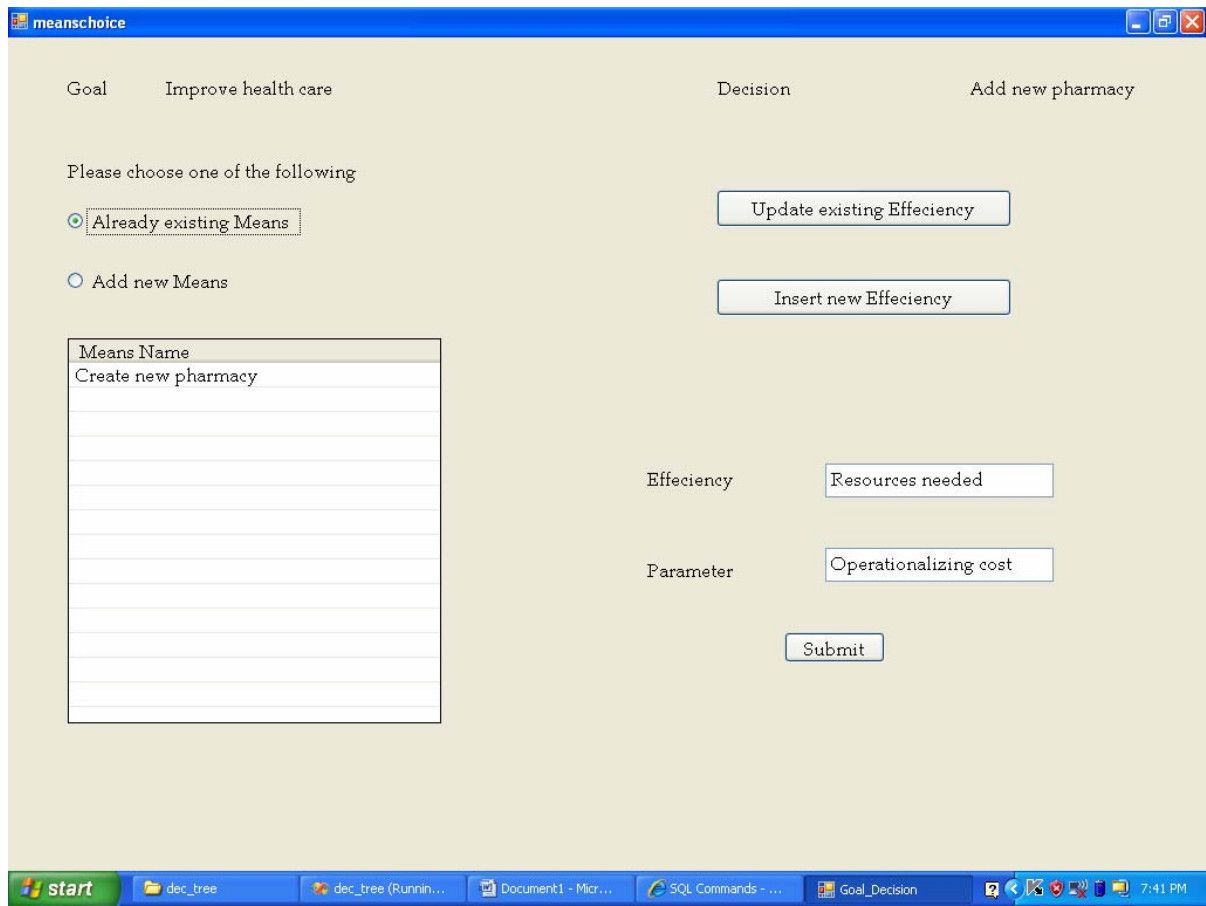

Fig 6. Obtaining Decision Parameters through Means Analysis

Fig. 6 shows an example of means analysis for the decision Add new pharmacy. It is possible to cerate the new pharmacy by splitting the existing pharmacy into two or by creating an altogether new pharmacy. The figure shows the second of these means to add a new pharmacy. The efficiency of the means can be evaluated by the efficiency criteria, resources needed and a parameter for this efficiency measure is operational zing cost.

\subsubsection{Outcome Feedback Analysis}

Sterman (Ste89) noted the crucial role played by outcome feedback and changes in the environment in management behaviour. Once a decision is taken, it produces an outcome. This outcome changes the environment and eventually feeds back into the outcome of the decision forming a feedback cycle. It follows that outcome feedback can help in discovering decision parameters by considering the environmental effects that a decision produces.

We illustrate the manner in which Raju supports outcome feedback analysis. Consider the decision Add new pharmacy once again. Addition of a new pharmacy shall affect the perception and behavior of the dispensary in which it is added. For example, there may be an increase in the registered members of the health service, which may lead to requirement of additional medical staff which in turn affects the pharmacies of the dispensary. Thus, we find a feedback cycle which starts off from the outcome of the decision, goes through the organization and returns back to the outcome. The screen of Fig. 7 shows this situation. 


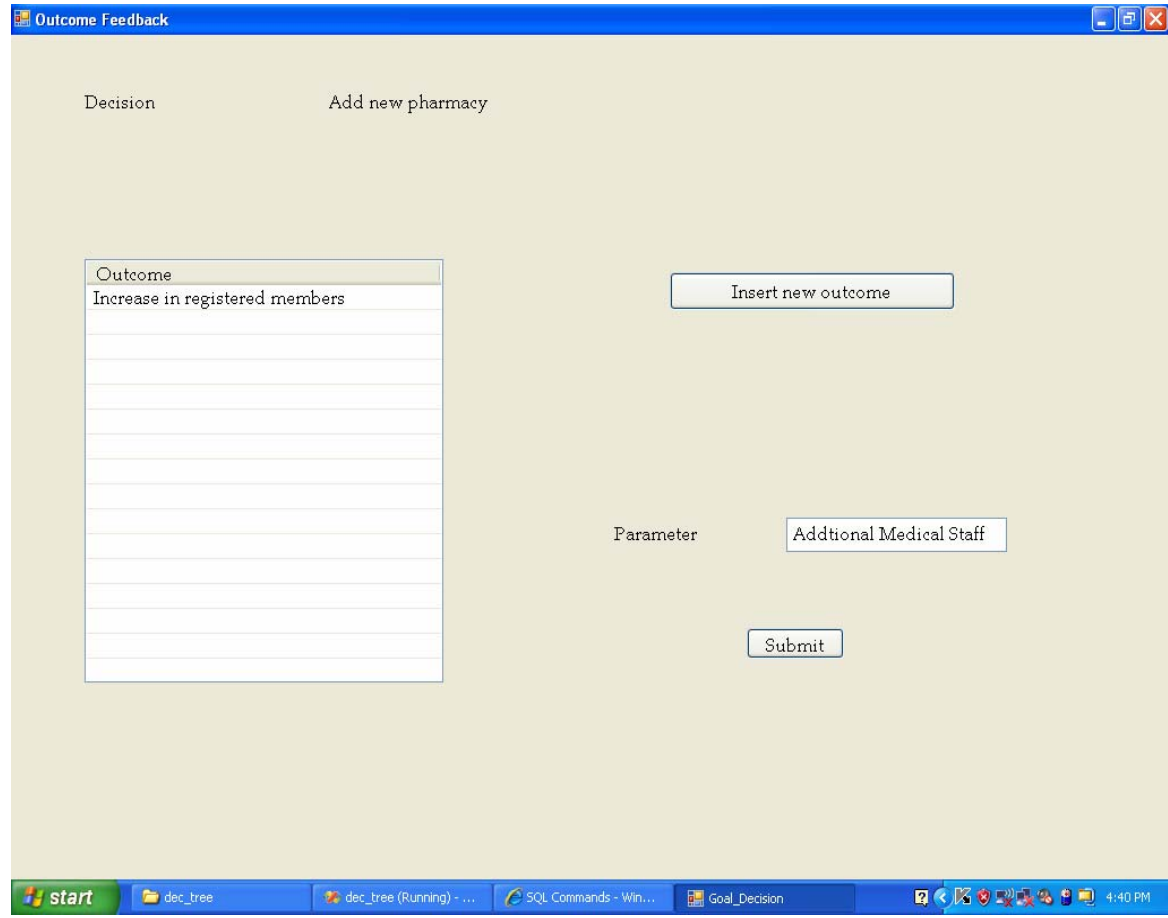

Fig. 7. Eliciting Parameters through Outcome Feedback Analysis

In the foregoing example we have one outcome that causes the feedback loop to close by reaching Add new pharmacy. In general, it is possible that a number of outcomes may be gone through before the starting decision is reached. Raju allows for this by allowing a number of outcomes to be entered. The one whose parameters are to be defined is selected and the parameters entered in the box on the screen. The closure of the feedback loop, that is, the outcome Add new pharmacy, in our example, is not entered in the outcome list.

\subsection{Information Identifier}

Raju uses heuristics to determine whether a parameter obtained through the Decision Parameter Guide is information or decision. These are as follows.

1. A parameter whose history is to be maintained. For example, registered members for the last five years. The history of the parameter registered members must be available for the last 5 years.

2. A parameter expressed category-wise. This category can be formed on temporal basis, for example, month-wise or on some semantic criterion like age wise, designation-wise etc.

3. A parameter that is a report or document.

4. A parameter that is obtained by applying a function like Count of, Average etc. 
5. A parameter involving comparison. For example, comparison of our performance against others.

The example in Fig. 8 is for the parameter, number of patients serviced. It is seen that it is a report, its history is to be maintained, and the function max is to be applied. That is the report should contain a five year history of the maximum number patients serviced per year. Since this parameter satisfies more than one heuristic, it is information that is relevant to the decision with which the parameter, number of patients, is associated.

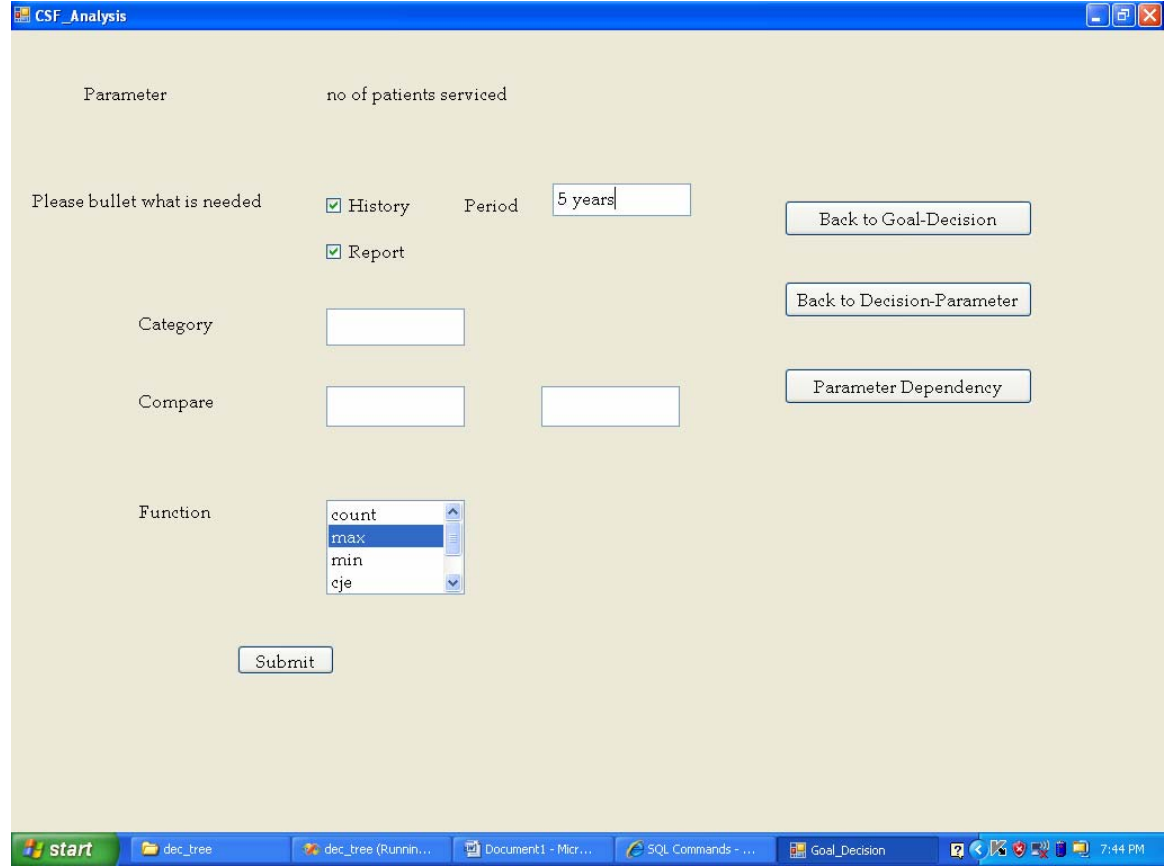

Fig. 8. Identifying Parameters as Information

\subsection{Sub Decision Identifier}

The sub decision identifier is charged with (a) determining which parameters are decisions, (b) determining the interrelationships between these newly postulated decisions and (c) applying our requirements engineering process of parameter elicitation etc. to each of these decisions.

Assume that $\mathrm{P}$ parameters were elicited for a decision and out of these I parameters are identified as information. Then (P-I) parameters are decision candidates. These (PI) decisions may be related to one another. The requirements engineer elicits these relationships through a dependency graph. The dependency graph represents which decision is dependent upon which other one. As an example consider the Add new pharmacy decision once again. Let the parameters $\mathrm{P}$ for this decision be 
$\mathrm{P}=\{$ waiting time of patients, operationalizing time, operationalizing cost, change in patients serviced, additional medical staff, change in registration, amount of medicine disbursed, patient serving capacity, average waiting time

Let the parameters I identified as information be

$\mathrm{I}=\{$ waiting time of patients serviced, operationalizing time, operationalizing cost, change in patients serviced, change in registration, amount of medicine disbursed, average waiting time\}

Therefore, the parameters that are decisions, D, are

$\mathrm{D}=\{$ additional medical staff, patient serving capacity $\}$

The parameters contained in D are shown on the left hand side of the screen of Fig. 9. Now, the requirements engineer draws a dependency graph on the right hand side of the screen by dragging and dropping the parameters. This dependency graph shows the decisions that are dependent on one another. Thus, starting from Add new pharmacy a hierarchy of decisions is constructed by the Sub Decision Identifier that shows which decision is the sub decision of which one. This hierarchy is stored in the repository. The newly discovered decisions are now sent through the requirements engineering process recursively till no new decisions are left.

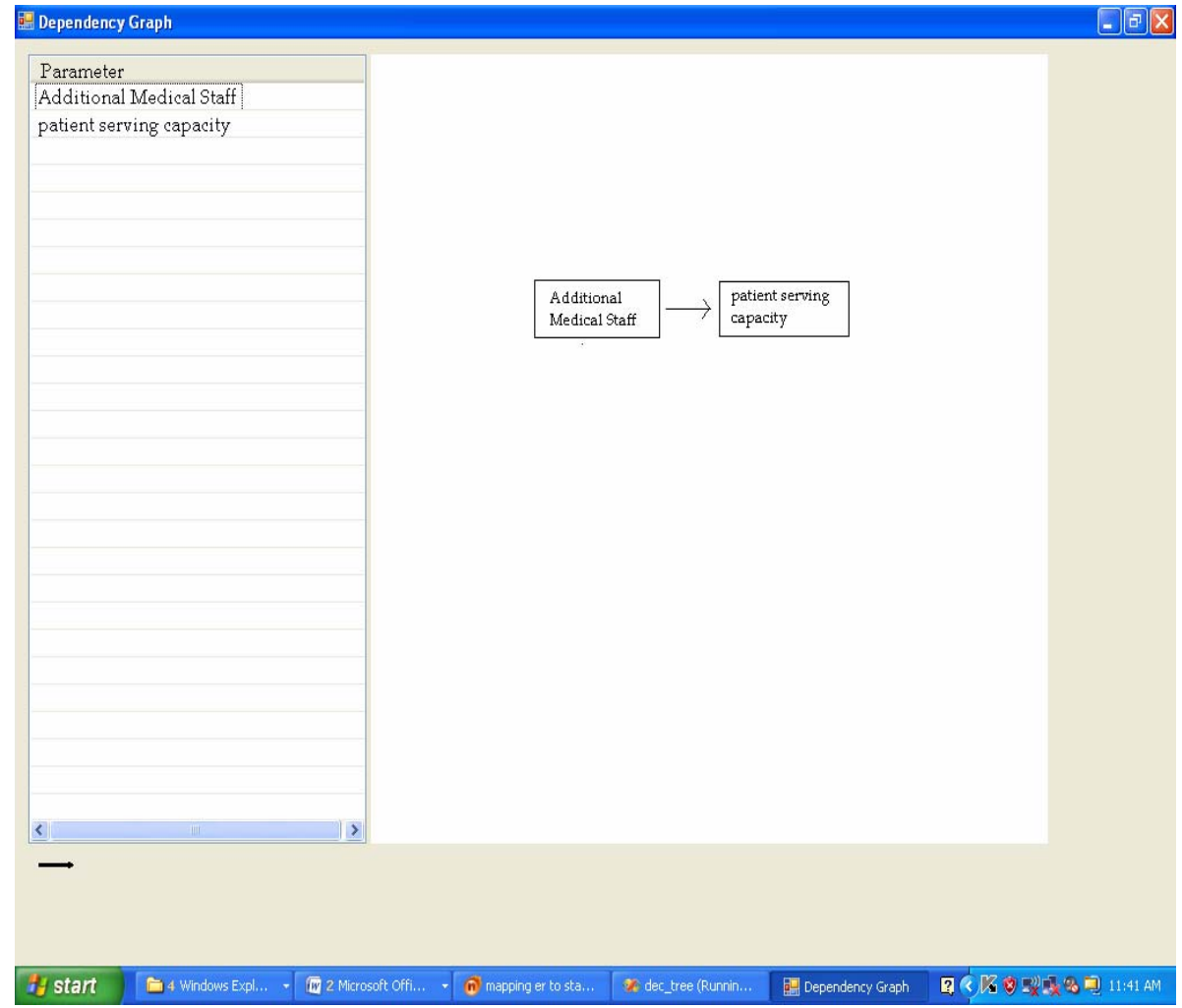

Fig. 9. Building the dependency graph 


\section{Impact: Comparison}

A comparison of our approach with goal oriented techniques in requirements engineering has been made in (Pra08). Here we compare our approach to DW development with other prevalent approaches. A detailed description of this is available in (Pra09).

An operational database for a health organization was available to us. This organization runs a number of dispensaries all over the country. The database maintains data about each dispensary of the health scheme. To avail of health services in a dispensary, one must be registered in the health scheme and then opt for the most convenient dispensary. Information about the registered members in the health scheme as well as in the dispensary is maintained in the database. Additionally, the database keeps track of the doctors, pharmacies, laboratories and other facilities of the dispensary. Each visit of the registered member, the disease, diagnosis, prescriptions, recommended tests and their results etc. are also kept track of. Inventory information of medicines, $\mathrm{X}$-ray films and other consumable and non-consumable items is maintained. When any of these gets depleted, it is indented from recognized chemists and the central store of the health scheme.

\section{The Golfarelli (Gol99) life cycle: Start development with database schema.}

This scheme assumes that the data contents of the operational databases more or less define the information contents of the data warehouse system to be built. Thus, a conversion of these into star/snowflake schemas is the major activity to be carried out. The basic idea is to examine each relation defined in the database(s) and identify whether it is a fact or a dimension. We believe that due to this, the technique is limited to the information in the database and identification of external sources and any other internal sources is difficult.

We used Oracle SQL Developer and Data Modeling tool Version: 1.5.1 (Ora) for developing the star schema of the health schema. The tool asked us to manually identify the relations that are facts and those that are dimensions. The dimension hierarchies and hierarchy levels were also to be specified manually. Based on this input, the tool created a snowflake schema. In applying this technique, we were not able to find any significant guidance on how to decide which relation is a fact or a dimension. Experience in applying the basic definitions of facts and dimensions available in the area of data warehousing, is essential.

Now consider deciding what historical data is to be maintained. Again, where temporal information is available in the operational database, one can assume that historical information shall also be needed in the data warehouse environment. For example, in our database, the date when a patient was examined is available. However, such temporal data was not available for indenting of depleted material. We needed to know if this is to be included in the data warehouse for projecting inventory requirements or not. Again this is ad-hoc and experience based and no guidance was available to us. Similarly, we found the definition of aggregates to be ad-hoc and experience based.

Involving stakeholders to look at every relation to identify facts is certainly possible but, in the absence of guidance, it is very ad-hoc and unsystematic. Stakeholders who are decision makers in an organization are not used to thinking in terms of relations and their mappings to data warehouse structures. 
Finally, there is no articulation of the decision capability supported. Thus, if a decision maker wants to know what decisions are supported or whether a given decision is supported then it is not possible to do so. This is because no relationship is established between the data and the decision for which it might be relevant.

\section{The Hüseman life cycle (Hüs00) that uses the ER schema}

The ER diagram of our health schema was not available to us. Therefore, we developed it from the documentation of the health scheme and the relational schema of the operational database. The requirements engineering problem is centered round the conversion of the ER schema into star/snowflake schemas.

Again, as for the database oriented approach, the assumption is that data warehouse contents are more or less available in the ER diagrams. Again, due to this, the technique is limited to the information in the ER schemas and identification of external sources and any other internal sources is difficult.

Regarding maintaining history it is to be noted that the ER schema does not specifically cater to temporal information and the real focus is on entities, relationships and attributes. This was true about our health scheme ER diagram as well which does not contain any temporal information. Therefore, deciding on whether or not historical data is to be kept was difficult in the absence of any guideline. Again deciding on aggregation of data from the ER diagram is hard in the absence of any guidelines to do so. There is no provision in the ER technique that makes its developer examine aggregates. Though one can define derived attributes, summarized information as required in data warehouses is not systematically investigated in this approach.

Lastly, there is no effort made in relating the ER diagram to the decision making capability to be provided. Therefore, it is not possible to know whether information for a given decision could be found in the data warehouse or not. In other words, it is not possible to know the decision capability supported by the data warehouse.

\section{The Goal-Decision-Information Approach}

Now, let us consider the approach adopted in this paper and the use of Raju. This approach distinguishes between transactions/functions that are required for operational systems and the managerial decisions to be taken. Identification of these managerial decisions is outside the scope of the work reported here. In terms of Fig. 2, this identification refers to the upstream activities of decision and goal elicitation respectively.

For the health scheme example, we arrived at goals and decisions through an informal interview process and from the health scheme documentation available with us. Our experience is that this is a non-trivial exercise and appropriate elicitation techniques for this have to be developed.

The initial set of goals and decisions were entered into Raju. Clearly, the identified information is obtained in an unstructured form. No effort is made to determine facts and dimensions or to move to the star/snowflake schema. The focus in Raju is on information elicitation rather than on information structuring. As a result, the thought process of the requirements engineer is channeled and the requirements engineering team is made to focus on the task at hand.

There is a close relationship between decisions and the information relevant to these. Additionally, since Raju starts off with an initial set of decisions, the decision 
making capability is well defined and it is possible to know what is and what is not supported by a data warehouse.

The information obtained may be unstructured but it is possible to identify its properties through Raju. Thus, it is possible to identify historical data and the number of years of history required as well as aggregates. This became quite evident in the health scheme example and only reinforced the use of heuristics of Fig. 8.

\section{Conclusion}

There is marked difference between the fitment issue in data warehouse systems and traditional information systems. This difference arises because the former are oriented towards decision making done by managers whereas the latter support transactions/functions performed by 'end users'. To do rational decision making, managers need information about the state of the organization and expectations of various stakeholders. The identification of this information is therefore a crucial issue. Poorly identified information leads to data warehouses that do not meet the requirements of managers. In contrast, when support is to be provided for transactions/functions then the identification of these functions is crucial. Additionally, the process model should fit well with that of the organization.

We have shown in this paper that the focus of database driven and ER driven life cycles seems to be not so much on information identification as on determination of facts and dimensions. Indeed this focus is very pronounced and even the determination of historical and aggregated information is not easily supported by these life cycles. In other words, interest is in determining the structure of the star/snowflake schemata from given information. This begs the issue of identification of needed information.

The support provided by Raju for the GDI approach elicits information relevant to decisions of interest to managers. This information is broad, top-level but has the property that aggregates and historical information can be identified. An additional step of conversion to data warehouse schemata is necessary. Our assumption is that this can be done using the notions of information scenarios and SSQL reported in [Pra08].

This leaves us with investigating the upstream activities, decision elicitation and goal elicitation parts of Fig. 2. We have started investigating the decision elicitation part.

\section{References}

[Go199] Golfarelli, M., Rizzi, S.: Designing the Data Warehouse: Key Steps and Crucial Issues. Journal of Computer Science and Information Management 2(3) (1999)

[Hüs00] Hüsemann, B., Lechtenbörger, J., Vossen, G.: Conceptual Data Warehouse Design. In: Proceedings of the International Workshop on Design and Management of Data Warehouses (DMDW 2000) Stockholm, Sweden, pp. 5-6 (2000)

[Mar91] James, G.: How decisions happen in organization. Human-Computer Interaction 6(2), 95-117 (1991) 
[Mon86] Montazemi, A.R., Conrath, D.W.: The Use of Cognitive Mapping for Information Requirements Analysis. MIS Quarterly 10(1), 45-56 (1986)

[Ora], http: / /www.oracle.com/technology/products/database/ sql_developer

[Pra04] Prakash, N., Singh, Y., Gosain, A.: Informational Scenarios for Data Warehouse Requirements Specification. In: Atzeni, P., Chu, W., Lu, H., Zhou, S., Ling, T.-W. (eds.) ER 2004. LNCS, vol. 3288, pp. 205-216. Springer, Heidelberg (2004)

[Pra08] Prakash, N., Gosain, A.: An Approach to Engineering the Requirements of Data Warehouses. Requirements Engineering Journal 13(1), 49-72 (2008)

[Pra09] Prakash, D.: Goal Oriented Requirements Engineering for Data Warehousing, M.Tech. thesis, IIIT Bangalore (2009)

[Ste89] Sterman, J.D.: Modeling Managerial Behaviour: Misperceptions of Feedback in a Dynamic Decision Making Experiment. Management Science 35(3), 321-339 (1989)

[Wet91] Wetherbe, J.C.: Executive Information Requirements: Getting it Right. MIS Quarterly 15(1), 51-65 (1991) 